\title{
Metástase de coróide simulando síndrome de efusão uveal: relato de caso
}

\author{
Choroidal metastasis simulating uveal effusion syndrome: case report
}

\author{
Carlos Eduardo Cury Júnior ${ }^{1}$ \\ Alexandre Cury Júnior ${ }^{2}$ \\ Rubens Camargosiqueira ${ }^{3}$ \\ Cíntia Storti ${ }^{4}$
}

\begin{tabular}{|l|}
\hline RESUMO \\
\hline Descrição de um caso de efusão uveal secundária a metástase de coróide \\
e revisão dos principais diagnósticos diferenciais. Uma paciente com \\
baixa da acuidade visual, no olho esquerdo, apresentou nódulos ao nível \\
da coróide, dispersão pigmentar retiniana e descolamento seroso da \\
retina. O exame angiofluoresceinográfico demonstrou padrão "leopard \\
spots", semelhante ao encontrado nos casos de efusão uveal. A ecografia \\
ocular demonstrou a presença do descolamento seroso da retina, presen- \\
ça de espessamento e nódulos na coróide e a tomografia de coerência \\
óptica evidenciou ruptura do epitélio pigmentado da retina, presença de \\
nodulações coroidianas hiperrefletivas e áreas de descolamento de \\
retina seroso. Após ser submetido a exames diagnósticos de imagem, \\
constatou-se a presença de nódulo único no ápice pulmonar esquerdo. \\
As metástases de coróide podem levar a um quadro similar à síndrome \\
de efusão uveal. Esta possibilidade deve sempre ser pensada diante de \\
um quadro de efusão uveal atípica e avaliação sistêmica para pesquisa de \\
carcinoma deverá ser solicitada.
\end{tabular}

Descritores: Neoplasias da coróide/secundário; Metástase neoplásica; Doenças da úvea; Úvea/patologia; Relatos de casos [Tipo de publicação]

\section{INTRODUÇ̃̃̃O}

A síndrome de efusão uveal pode ser ocasionada por condições inflamatórias, espessamento escleral idiopático ou nos casos de nanoftalmia e tumores primários ou metastáticos. O carcinoma metastático da coróide é reconhecidamente a neoplasia intra-ocular mais comum ${ }^{(1-7)}$. Em contraste, metástases limitadas à retina e ao nervo óptico são infreqüentes e apenas alguns relatos na literatura são descritos ${ }^{(4)}$. Os sítios primários mais comuns do tumor são, em ordem decrescente: mama em $47 \%$ dos casos, pulmão em $21 \%$ e trato gastrointestinal em $4 \%$. Nos casos de carcinoma metastático da coróide, secundários a tumor de mama, 90\% dos pacientes apresentam história de tumor, previamente ao diagnóstico ocular ${ }^{(8)}$. Entretanto, nos pacientes com sítio primário desconhecido, o câncer de pulmão é o mais comum tanto no sexo masculino quanto no feminino ${ }^{(8)}$. Neste relato nós descrevemos uma paciente de 65 anos com diagnóstico de carcinoma metastático da coróide simulando um quadro de síndrome de efusão uveal.

\section{RELATO DE CASO}

Paciente de 65 anos do sexo feminino com história de baixa da acuidade visual progressiva no olho esquerdo há 8 meses. A paciente negava antece- 
dentes familiares e apresentava, como antecedentes patológicos, cardiopatia chagásica, hipertensão arterial sistêmica, histórico de tabagismo durante 30 anos. Ao exame oftalmológico, a acuidade visual com a melhor correção era 20/20 em olho direito e contar dedos a 50 centímetros em olho esquerdo, ausência de defeito pupilar aferente e tonometria de aplanação de $15 \mathrm{mmHg}$ em ambos os olhos. A fundoscopia do olho direito não apresentava alterações e o olho esquerdo apresentava quadro de: dispersão pigmentar pela retina, nodulações difusas ao nível da coróide, descolamento seroso da retina (DRS) e edema do disco óptico (Figura 1). O corpo vítreo apresentava-se acelular em ambos os olhos. O exame angiofluoresceinográfico demonstrou aspecto tigróide, tipo "leopard spots", com áreas de hipofluorescência por bloqueio pigmentar permeadas por áreas de hiperfluorescência transmitida. O disco óptico, na fase inicial, apresentava extravasamento do contraste o qual acentuouse nas fases mais tardias (Figura 2). A ecografia ocular no modo A e B demonstrou a presença de imagens hiperecogênicas ao nível da coróide com média refletividade interna (Figura 3). A tomografia de coerência óptica (OCT) revelou imagens hiperrefletivas ao nível da coróide, nos locais onde haviam nodulações à fundoscopia, ruptura do epitélio pig- mentado da retina (EPR) e áreas de hiporrefletividade subretinianas compatíveis com o DRS (Figura 4). Solicitamos avaliação sistêmica para a detecção de neoplasia primária ou de doença granulomatosa. O exame físico realizado apresentouse normal. Os exames laboratoriais solicitados, tais como: hemograma, coagulograma, Enzima conversora da angiotensina (ECA) e sorologia para sífilis, não revelaram nenhuma alteração. Foram solicitados exames radiológicos como: radiografia de tórax, que revelou a presença de imagem radiopaca ao nível do ápice pulmonar esquerdo e aumento da área cardíaca (Figura 5A), mamografia sem alterações e ressonância nuclear magnética torácica demonstrando a presença de lesão tumoral no parênquima pulmonar, contínua com a pleura, na região do ápice pulmonar esquerdo (Figura 5B e C).

\section{COMENTÁRIOS}

A efusão uveal pode ocorrer como resultado de processo inflamatório, anormalidades esclerais ou aumento da pressão na circulação coroidal ${ }^{(1)}$. Alguns autores propuseram que o descolamento cilio-coroidal seria causado por hipotonia, inflamação intra-ocular e/ou tumores intra-ocu-
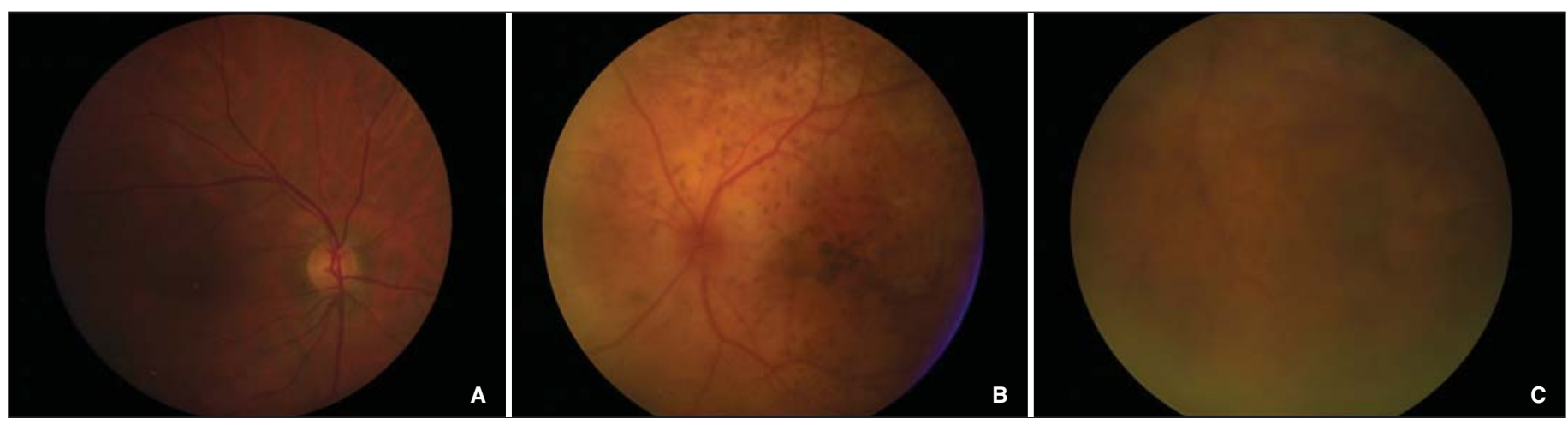

Figura 1 - A: Retinografia colorida do olho direito sem alterações; B e C: Retinografia colorida do olho esquerdo demonstrando alterações difusas do EPR e presença de nódulos sub-retinianos
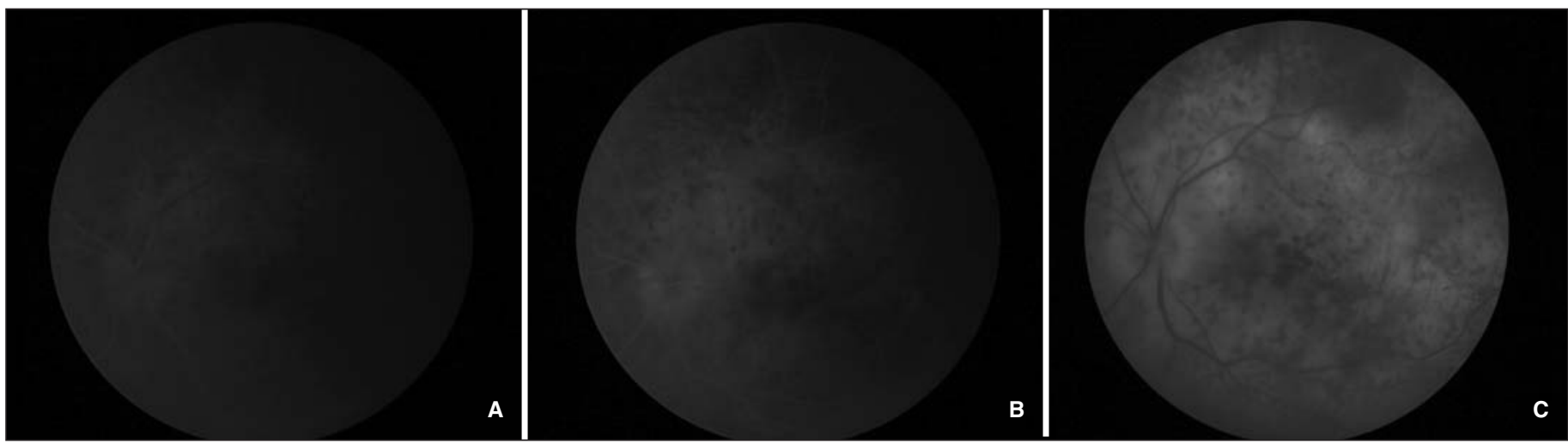

Figura 2 - Retinografia fluorescente do olho esquerdo. A: Fase inicial; B: Fase intermediária demonstrando áreas de hipofluorescência por bloqueio e hiperfluorescência no disco óptico pelo extravasamento do contraste; C: Fase tardia demonstrando hiperfluorescência com áreas de hipofluorescência aspecto tigróide tipo "leopard spots" e extravasamento do contraste no disco óptico. 
lares ${ }^{(9)}$. Estes devem ser sempre lembrados, como diagnóstico diferencial, nos casos de síndrome de efusão uveal secundária. Neste caso em questão, relatamos um paciente com quadro similar à efusão uveal secundário a carcinoma metastático da coróide, porém sem apresentar alterações ecográficas compatíveis com a síndrome. Um relato de caso $^{(10)}$ demonstrou a manifestação de um carcinoma metastático da coróide como efusão uveal, acompanhada dos sinais clássicos como descolamento da coróide, ao exame ecográfico, e "leopard spots". A metástase uveal é o tumor intra-ocular mais comum ${ }^{(2-4)}$, sendo a coróide o local mais acometido. O diagnóstico do carcinoma metastático da coróide é desafiador, quando se desconhece a presença de um tumor primário ${ }^{(8)}$. Esses autores demonstraram, em um estudo, que o foco primário já era conhecido antes do diagnóstico de carcinoma metastático da coróide em $66 \%$ dos casos. Dos 34\% restantes, o sítio primário foi identificado em $49 \%$, sendo o pulmão responsável por $70 \%$ destes $\operatorname{casos}^{(8)}$. O exame fundoscópico, do olho esquerdo, demonstrava a presença múltiplos nódulos na coróide, descolamento seroso da retina e alterações no EPR. Vários tipos de lesões podem simular o carcinoma metastático da coróide, tais como: melanoma de coróide, osteoma de coróide, hemangioma de coróide, neovascularização da coróide com cicatriz disciforme e sarcoidose. O envolvimento do segmento posterior por sarcoidose ocorre em $25 \%$ dos casos e geralmente se manifesta como coriorretinite multifocal e periflebite retiniana ${ }^{(8,11)}$. Outra forma, menos comum, é o granuloma solitário de coróide ${ }^{(12)}$. Situações inflamatórias como: doença de Harada, síndrome da efusão uveal idiopática e esclerite posterior também devem ser incluídas no "pool” de diagnósticos diferenciais. Nestes casos inflamatórios geralmente há presença vitreíte e es-

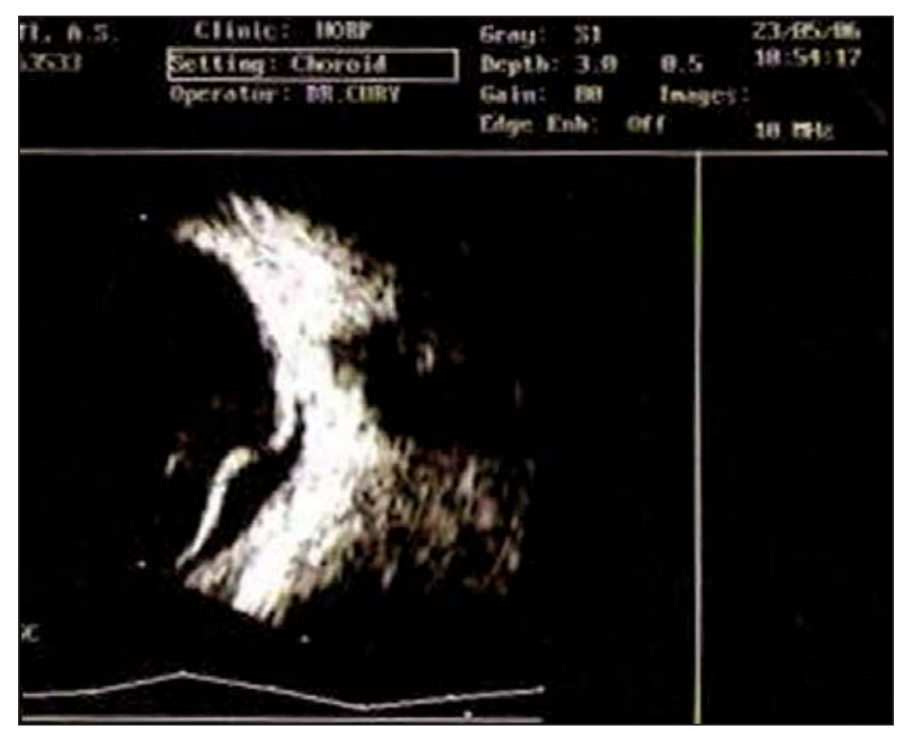

Figura 3 - Ecografia ocular (modo B) do olho esquerdo demonstrando o descolamento seroso da retina, espessamento e nódulos na coróide com refletividade interna moderada (seta branca) pessamento da coróide, observado ao exame ecográfico. Neste caso, o padrão "leopard spots", típico da síndrome da efusão uveal, foi encontrado no exame angiofluoresceinográfico. O exame ecográfico revelou DRS inferior, espessamento de coróide e presença de nódulos de média refletividade interna. O carcinoma metastático da coróide, secundário ao câncer de mama, geralmente se apresenta como uma ou mais lesões sésseis, com pouca elevação e infiltrados amarelados. Os casos de carcinoma metastático da coróide por câncer pulmonar podem ter estas características porém, mais freqüentemente, as lesões são mais elevadas, cupuliformes e semelhantes ao melanoma primário da coróide $^{(13)}$. No caso em questão, observamos uma apresentação menos comum, do carcinoma metastático da coróide por tumor pulmonar, com múltiplos nódulos coroidianos. Em um estudo realizado em 520 olhos a distribuição em relação ao sítio primário do carcinoma metastático da coróide, no sexo feminino foi: mama em $67 \%$, pulmão $12 \%$, desconhecido em $12 \%$, gastrointestinal em $2 \%$ e pele em $1 \%$ dos casos. Estudos clínicos e histopatológicos de pacientes com carcinoma metastático da coróide demonstram alterações secundárias que incluem: descolamento seroso da retina que geralmente extrapola os limites do tumor, atrofia do EPR e da retina, degeneração cistóide da retina levando a quadros de retinosquise e invasão da retina neurosensorial pelo tumor ${ }^{(8,14-15)}$. O OCT pode mostrar claramente as alterações estruturais na retina, que circunda o tumor de coróide, embora não forneça nenhuma informação sobre sua histopatologia ${ }^{(16)}$. Autores demonstraram, através de
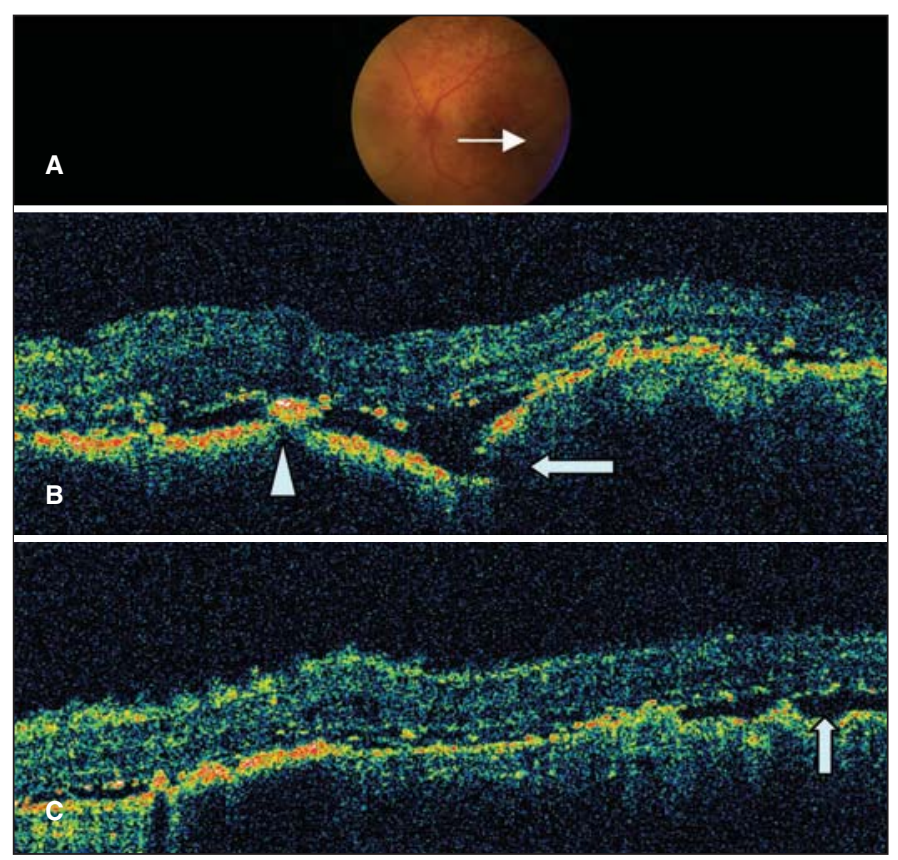

Figura 4 - Tomografia de coerência óptica (OCT). A: Retinografia colorida do olho esquerdo demonstrando a orientação do scaneamento; B: Rotura do EPR (seta branca) e nódulo hiperrefletivo ao nível do complexo EPR-córiocapilar (cabeça da seta); C: Área hiporrefletiva correspondente ao descolamento seroso da retina (seta) 

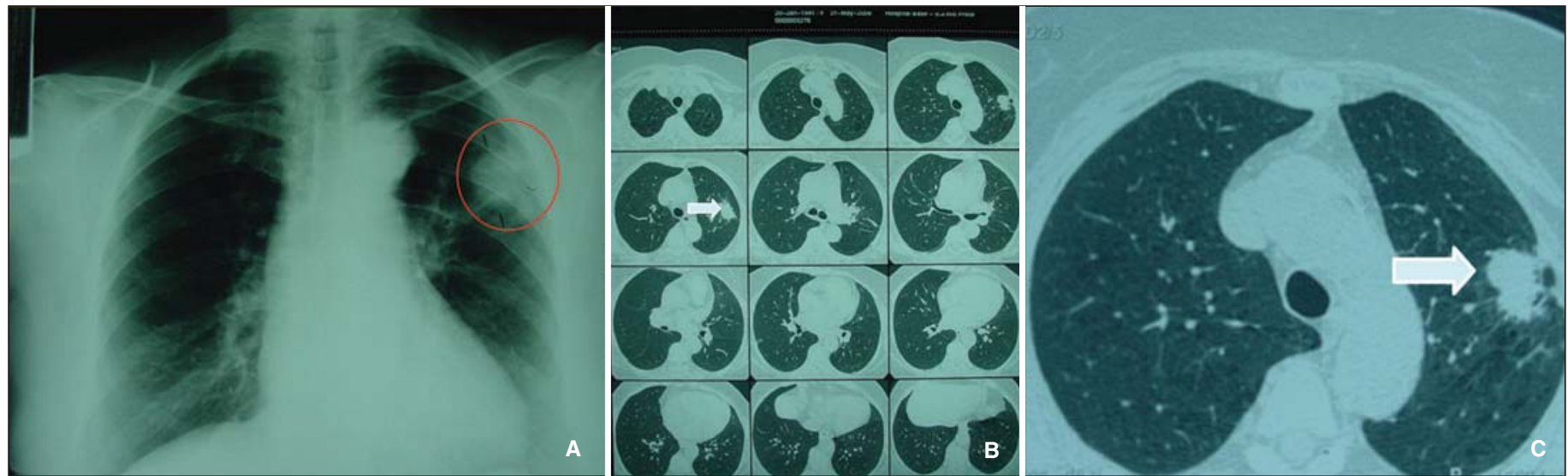

Figura 5 - A: Rx de Tórax demonstrando aumento da área cardíaca e presenca de nódulo pulmonar solitário no ápice pulmonar à esquerda (círculo); B e C: Tomografia computadorizada de tórax demonstra a nodulação pulmonar e na imagem C a continuidade com a pleura

estudo tomográfico em 7 olhos com carcinoma metastático da coróide, alterações características da retina e do complexo EPR-córiocapilar ${ }^{(15)}$. Os achados mais encontrados foram áreas de hiporrefletividade correspondentes ao DR seroso e lesões hiperrefletivas sobre o complexo EPR-córiocapilar em $87,5 \%$ dos casos. Neste caso o OCT demonstrou a presença de rotura do EPR, além de algumas lesões descritas neste estudo. Através deste relato concluímos que, o papel do oftalmologista é primordial, em algumas situações, no diagnóstico de tumores sistêmicos que se apresentam como metástase coroidiana. Na presença de quadro clínicoangiográfico, semelhante ao da efusão uveal, deve-se afastar a hipótese de carcinoma metastático da coróide.

\section{ABSTRACT}

We report a case of choroidal metastasis simulating uveal effusion syndrome. This patient with uveal metastasis from lung carcinoma presented with decreased vision in the left eye. Fundoscopic examination revealed pigmentary clumping, multiple choroidal nodules and serous retinal detachment. Fluorescein angioraphy revealed a leopard spots pattern similar to uveal effusion syndrome. Ultrasound revealed the presence of choroidal nodules with moderate internal reflectivity, choroidal thickening and serous retinal detachment. Optical coherence tomography showed retinal pigment epithelium tear and hyper-reflective nodules within the choroid and areas of serous retinal detachment. Image investigation demonstrated the presence of a solitary pulmonary nodule at the left apical lung. Choroidal metastasis can lead to a scenario that simulates uveal effusion syndrome. This possibility must be considered when having an atypical uveal effusion symptomatoloy and a systemic screening for systemic cancer should be performed.
Keywords: Choroid neoplasms/secondary; Neoplasm metastasis; Uveal diseases; Uvea/pathology; Case reports [Publication type]

\section{REFERÊNCIAS}

1. Duke JR, Walsh FB. Metastatic carcinoma to the retina. Am J Ophthalmol. 1959;47(1 Pt 1):44-8.

2. Koenig RP, Johnson DL, Monahan RH. Bronchogenic carcinoma with metastases to the retina. Am J Ophthalmol. 1963;56:827-9.

3. Leys AM, Van Eyck LM, Nuttin BJ, Pauwels PA, Delabie JM, Libert JA. Metastatic carcinoma to the retina. Clinicopathologic findings in two cases. Arch Ophthalmol. 1990;108(10):1448-52.

4. Shields JA, Shields CL. Intraocular tumors: a text and atlas. Philadelphia: WB Saunders; 1992. p.207-38.

5. Corrêa ZMS, Irion LCD, Marcon IM, Goldhardt R, Travi GM. O diagnóstico do carcinoma metastático de coróide pela biópsia aspirativa com agulha fina (BAAF) - relato de caso. Arq Bras Oftalmol. 2003;66(2):213-8.

6. Oliveira AA, Oréfice F, Miranda D. Carcinoma metastático na coróide simulando doença de Harada. Arq Bras Oftalmol. 1983;46(2):31-5.

7. Farias CC, Garcia CR, Muccioli C. Síndrome mascarada como primeira manifestação de adenocarcinoma pulmonar: relato de caso e revisão de literatura. Arq Bras Oftalmol. 2003;66(2):231-4.

8. Shields CL, Shields JA, Gross NE, Schwartz GP, Lally SE. Survey of 520 eyes with uveal metastases. Ophthalmology. 1997;104(8):1265-76.

9. Gass JD, Jallow S. Idiopathic serous detachment of the choroid, ciliary body, and retina (uveal effusion syndrome). Ophthalmology. 1982;89(9):1018-32.

10. Kreiger AE, Meyer D, Smith TR, Riemer K. Metastatic carcinoma to the choroid with choroidal detachment. A case presenting as uveal effusion. Arch Ophthalmol. 1969;82(2):209-13.

11. Obenauf CD, Shaw HE, Sydnor CF, Klintworh GK. Sarcoidosis and its ophthalmic manifestations. Am J Ophthalmol. 1978;86(5):648-55.

12. Desai UR, Tawansy KA, Joondeph BC, Schiffman RM. Choroidal granulomas in systemic sarcoidosis. Retina. 2001;21(1):40-7.

13. Shields JA, Shields CL. Clinical features of posterior uveal melanoma. In: Shields JA, Shields CL, editors. Atlas of intraocular tumors. Philadelphia: Lippincott Williams \& Wilkins; 1999. p.74-93.

14. Damato BE, Foulds WS. Tumour-associated retinal pigment epitheliopathy. Eye. 1990;4(Pt 2):382-7.

15. Arevalo JF, Fernandez CF, Garcia RA. Optical coherence tomography characteristics of choroidal metastasis. Ophthalmology. 2005;112(9):1612-9.

16. Schaudig U, Hassenstein A, Bernd A, Walter A, Richard G. Limitations of imaging choroidal tumors in vivo by optical coherence tomography. Graefes Arch Clin Exp Ophthalmol. 1998;236(8):588-92. 